Perspectiva de Familia (Perspect. Fam.) ISSN 2415-5187 (impreso), ISSN 2519-1748 (en línea), año 2019, vol. 4, pp. 67-83

\title{
Libertad moral: consecuencia del desarrollo de virtudes
}

Moral freedom: a consequence of virtues development

María L. Diez Canseco Briceño

Universidad Católica San Pablo, Perú

Correspondencia: ldiezcanseco@ucsp.edu.pe

Recibido: 13-08-2019. Revisado: 18-09-2019. Aceptado: 07-10-2019

Citarlo como: Diez Canseco, M. L. (2019). Libertad moral: consecuencia del desarrollo de virtudes. Perspectiva de Familia, 4, pp. 67-83.

\section{Resumen}

El presente trabajo propone que la libertad humana es posible de ser educada por medio de la forja y desarrollo de virtudes morales. La premisa en la que se basa este planteamiento es que una auténtica libertad en el ser humano es la que responde a su misma naturaleza y a la verdad de las cosas. Esto será posible por medio del ejercicio de las virtudes morales, las cuales pueden y deben ser educadas, especialmente, en la familia, lugar por excelencia para que la persona humana alcance su mayor despliegue. A partir de la revisión bibliográfica, se puede concluir que la forja de hábitos virtuosos llevada a cabo desde edades tempranas posibilita que la persona alcance un conocimiento y gobierno personal que le permita hacer un ejercicio de su libertad que lo lleve al bien.

Palabras clave: Libertad, moral, virtudes, educación.

\begin{abstract}
The present work proposes that human freedom is possible to be educated through the forging and development of moral virtues; the approach is based on the premise that an authentic freedom in the human being, is the one that responds to its
\end{abstract}


very nature and the truth of things and this will only be possible through the exercise of moral virtues, which can and should be educated in family, a place par excellence for the human person to achieve its greatest deployment. From the literature review it can be concluded that the forging of virtuous habits carried out from an early age, allows the person to reach a knowledge and personal government that allows him to exercise his freedom that leads him to good.

Key words: Freedom, moral, virtues, education.

En la actualidad, es muy frecuente que la noción de libertad sea entendida sin tener en cuenta su lazo con la verdad, con la estructura ontológica del hombre y la estructura de las mismas cosas (Elders, 1997). A partir de ello es que surgen distintas interpretaciones que se centran en entenderla como la posibilidad de actuar en función de las propias apetencias o deseos con una absoluta independencia (López, 1977).

Es bajo el sustento de estos criterios que, en la sociedad, se ven una serie de manifestaciones colectivas en donde se pretende imponer un derecho de las personas a ejercer su libertad, considerando que la misma radica en la posibilidad de poder decidir por aquello que cada uno determine como lo mejor según su circunstancia concreta. Dentro de algunos ejemplos de estas manifestaciones estaría el derecho de las mujeres al aborto, aduciendo que son dueñas de su cuerpo; el derecho de los medios de comunicación a emitir programas impropios, aduciendo la libertad de expresión, entre otros.

Bajo este contexto, se hace necesario llevar a cabo estudios que permitan tener una mayor claridad acerca de lo que significa realmente un auténtico ejercicio de la libertad en la persona humana. Es así que el presente análisis está basado en la propuesta de Santo Tomás de Aquino y de pensadores que han desarrollado a profundidad el tema de la libertad y de su educación tomándolo como punto de referencia: Servais Pinckaers, Josef Pieper, Antonio Millán Puelles, Antonio Amado, Enrique Martínez, Mercedes Palet, Martín Echavarría, entre otros.

La investigación efectuada consiste en una revisión teórica de lo planteado por Santo Tomás y los autores mencionados arriba. El objetivo del trabajo es, a partir de la revisión bibliográfica, ordenar los distintos contenidos referidos al tema de la libertad en pos del planteamiento de que la misma es posible de ser educada a través del desarrollo de virtudes.

En esta línea, es necesario hacer una reflexión que lleve a una mayor comprensión de la finalidad de la educación, ya que se podría decir que, en la actualidad, no hay 
un consenso sobre lo que significa educar y sus implicancias (Martínez, 2002). En la acción educativa, muchas veces se pierde de vista su horizonte y su finalidad, y se pone mayor énfasis en aquello que tiene que ver, sobre todo, con el perfeccionamiento del «hacer»y no con el perfeccionamiento del «ser» de la persona humana (Diez Canseco, 2018). Por eso, se busca desde ciertas posturas - como el conductismo- que el niño configure su comportamiento exterior (Barrio Maestre, 1998) en función del manejo que haga el educador de las contingencias ambientales.

Por otro lado, desde el constructivismo, la finalidad de la educación es que el niño cuente con las herramientas necesarias y suficientes para que construya sus conocimientos, debido a que se concibe al hombre como un ser carente de intimidad y subjetividad, cuya interioridad es acción y está orientada a la transformación exterior del mundo (Echavarría, 2012).

Estas maneras de entender la labor educativa dan lugar a que se busque un perfeccionamiento de las acciones del hombre y de un obrar que sale fuera de sí. En ese aspecto, no se considera el perfeccionamiento de la persona en su totalidad, sino solo de su obrar, y ello se debe a que existe una concepción equivocada sobre la moral y sobre el ser persona (Diez Canseco, 2018), dado que se asocia este concepto de moral «con la idea de una doctrina del hacer y, sobre todo, del no-hacer, del poder y no-poder, de lo mandado y lo prohibido» (Pieper, 2007, p. 12).

Tomando en cuenta lo planteado líneas arriba es que el presente trabajo busca abordar el tema de la libertad humana a partir de una comprensión de lo que significa e implica ser persona humana. En este sentido, una recta aproximación llevará a entender que la autonomía de la que goza la persona está basada en un orden previo propio de la misma naturaleza humana y también en un orden establecido desde fuera. Será a partir de ello que el ser humano tendrá la posibilidad de ejercer un pleno dominio sobre sus inclinaciones naturales y potencialidades gracias al ejercicio de su razón y gobierno voluntario de su accionar.

El ser humano requiere de una guía y conducción que lo ayude a ejercer un gobierno sobre sus tendencias afectivas que le permita tomar decisiones que lo lleven al bien, esto será posible en la medida que se adquieran virtudes, especialmente las morales (Diez Canseco, 2018). Es por esta razón que se propone que la libertad es posible de ser educada y, ante todo, que «debe» ser educada para el bien de la persona, puesto que los dinamismos que fundamentan sus acciones libres son el conocimiento de la realidad y los actos voluntariamente elegidos que lleven al bien.

El logro de la virtud moral da lugar a un perfeccionamiento de determinadas potencias (Echavarría, 2015), lo que permite que el ser humano pueda desplegarse según 
lo que su misma naturaleza le exige. Para que ello se pueda dar, es requisito que la persona sea sujeto de una acción educativa, que se debe brindar principalmente en el seno familiar, ya que la forja de la virtud se debe iniciar desde los primeros años de vida. En el presente trabajo, se proponen una serie de pasos a seguir y un orden para el logro de cada una de las virtudes morales, las que, al configurarse en una segunda naturaleza en la persona, darán como resultado la posibilidad de un actuar plenamente libre, a partir de un recto discernimiento sobre la realidad concreta y un gobierno personal que permite que la persona opte por el bien y por lo mejor.

\section{Consideraciones sobre libertad}

Brennan (1960) explica que en relación con la libertad del ser humano existen tres posturas deterministas, las cuales, de alguna manera, sostienen la imposibilidad de una acción libre por parte de la persona. En primer lugar, está el determinismo físico, que propone una causalidad necesaria en los eventos naturales, dándose una plena identificación entre lo físico y lo psicológico. Por otro lado, está el determinismo biológico, que plantea que toda conducta humana está regida por las inclinaciones naturales. Y, finalmente, el determinismo psicológico, que establece que cuando la voluntad actúa debe elegir el bien u objeto de mayor valor; en este caso, la voluntad se vería forzada a seguir un orden determinado de bienes.

Además, existen posturas en donde se considera que el ser humano es capaz de poseerse a sí mismo y, por ello, capaz de elegir libremente. Una de estas es la desarrollada por Pinckaers (2007), para quien existe una libertad de indiferencia y una libertad de calidad. La primera sugiere que el libre arbitrio es anterior a la razón y a la voluntad y, por lo tanto, es aquello que dirige a las mismas; de esta forma, la libertad es algo indiferente frente a dos opciones contrarias entre sí. Desde esta perspectiva, propuesta en un primer momento por San Buenaventura y posteriormente por Ockham y los nominalistas (Pinckaers, 2007), se piensa que la libertad se identifica con el mismo ser del hombre, puesto que es el origen de sus actos. Así mismo, esta libertad de indiferencia plantea un ideal de libertad en la que se da una decisión sin motivos (Verneaux, 1988), en donde la persona puede estar frente a motivos contrarios y no tiene que elegir el mejor, sino el que, en ese momento, le apetece; aquí se niega cualquier forma de dependencia del hombre hacia algo que no sea él mismo, es decir, una total autonomía frente a los actos aislados que lleva a cabo. Por esto, la fuente de esta concepción de libertad es «la voluntad en el hombre de la afirmación de sí en una radical diferencia respecto de lo que no es él mismo» (Pinckaers, 2007, p. 399).

Desde esta postura de libertad de indiferencia que Pinckaers presenta en su obra, podemos darnos cuenta de que la concepción que está detrás sobre la persona hu- 
mana considera que las inclinaciones naturales, la posibilidad de gobierno racional sobre las pasiones, la noción de que el hombre tiende hacia un fin último y es capaz de ordenar los medios necesarios para alcanzarlo y, en esa línea, la formación y desarrollo de virtudes es algo ajeno a la persona; y más bien se propone una afirmación total de la voluntad, aunque ello implique un distanciamiento entre esta y razón. Es aquí que surge la pregunta sobre la cuestión moral; si el hombre es un ser libre en la medida en que actúe en función de lo que su voluntad le indique sin que exista ninguna dependencia hacia sus inclinaciones naturales, ¿cómo es posible afirmar qué es lo moralmente correcto y qué no? En esta línea, la propuesta plantea que la voluntad divina manifestada por medio de la ley es la que somete la voluntad del hombre y se le impone como una obligación y un límite (Pinckaers, 2007).

Por otro lado, tenemos la voluntad de calidad (Pinckaers, 2007), en donde la concepción de libertad es vista desde otro ángulo. En primer lugar, se cree que la libertad no niega las inclinaciones naturales, por el contrario, se arraiga en ellas; en el hombre «el germen natural de la libertad está formado por el sentido de lo bueno, verdadero, de la rectitud, del amor, el deseo de conocimiento y de felicidad» (p. 421). La libertad, desde este punto de vista, consiste en el supuesto de un orden previo al cual el hombre responde y una dirección hacia la cual tiende, ello le permite actuar con total independencia y autonomía, pero en función de dicho orden, que es el que le da sentido a la existencia humana.

Como podemos ver, si nos basamos en la concepción de libertad de indiferencia, la posibilidad de educación de la libertad humana queda totalmente negada. Sin embargo, desde la perspectiva de la libertad de calidad sí es posible ordenar las inclinaciones naturales del hombre para que pueda alcanzar el fin hacia el cual está llamado y, en este marco, se hace necesaria una acción educativa. Por lo tanto, se puede afirmar que la libertad es posible de ser educada, pero no a través de la imposición de normas y reglas, sino mediante el cultivo de las inclinaciones naturales, para que el hombre pueda responder plenamente a ellas. La posibilidad de optar por el mal siempre está presente, lo cual corresponde a una deficiencia de la misma y no a un pleno despliegue de la libertad del hombre, ya que por naturaleza este tiende al bien (Ocampo, 2013), el cual es «lo conforme con la realidad» (Pieper, 1974, p. 15).

\section{Papel del conocimiento y la voluntad en la dinámica de la libertad humana}

El hecho de que el ser humano sea capaz de llevar a cabo acciones libres, en donde no se vea coaccionado por ninguna situación externa, implica que la persona es capaz de actuar basada en una deliberación acerca de la realidad. Esto debe dar lugar a una decisión personal acorde con el bien y la verdad, conocidos e internalizados 
y en correspondencia con la propia naturaleza humana; todo ello es posible en la medida en que la persona alcance cierta madurez y desarrollo. El camino para ello no es individual, sino más bien de acompañamiento por alguien que oriente y guíe; ese alguien, en principio, deben ser los padres, y el espacio por excelencia para que se dé la acción educativa, la familia (Palet, 2000).

El punto de partida para un ejercicio pleno de la libertad es la posibilidad de un recto conocimiento de la realidad, ya que «la potencia cognoscitiva mueve a la apetitiva presentándole su objeto» (Tomás de Aquino, 2001, I-II, q.40, a.2). Es así que luego del conocimiento de la realidad se da una tendencia hacia la misma y, por ende, la posibilidad de llevar a cabo una elección; «nuestro querer y obrar están determinados por el conocimiento» (Pieper, 1974, p. 21). En ese sentido, la primera acción educativa en aras de forjar una recta libertad en la persona va en la línea de procurar que esta tenga un adecuado conocimiento de la realidad; si dicho conocimiento es tergiversado o subjetivo, de alguna manera se verán empañadas sus tendencias y elecciones.

Bajo este criterio, para que el conocimiento que la persona tenga de la realidad sea acorde con la misma, se requiere del ejercicio de la razón, puesto que por medio de ella se lleva a cabo un discernimiento sobre los distintos bienes conocidos, lo que da lugar a la elección de aquel que se considera más completo y mejor. Es de esta forma que la libertad se «funda en la razón que sigue la voluntad» (Pinckaers, 2007, p. 459) y la voluntad, por su parte, al tener una inclinación hacia el fin o bien (Echavarría, 2016), consiste en un modo de posicionarse ante el bien abstracto que la inteligencia le muestra.

La acción voluntaria no se ve forzada a amar o desear determinados bienes particulares (Brennan, 1960), antes bien, la persona está en la capacidad de elegirlos o no, ya que dichos bienes son los medios para alcanzar el fin último, el cual no es posible de ser elegido. Santo Tomás lo plantea con mucha claridad: «no se elige el fin, sino lo que lleva al fin, como se dice en III Ethicorum. Por lo tanto, el deseo del último fin no es algo de lo que seamos dueños» (Tomás de Aquino, 2001, I-I, q.82, a.1, in c). Pinckaers (2007) nos hace ver que esto podría suponer una limitación de la libertad en el ser humano, pero en realidad es lo contrario, puesto que este conocimiento de la verdad y aspiración al bien abren un horizonte infinito a las posibilidades de elección del hombre para alcanzarlos.

En la elección de los bienes entendidos como medios para alcanzar el fin último, el conocimiento recto de la realidad cumple un papel muy importante. Por ello, el entendimiento debe mostrar a la voluntad la realidad tal y como es, porque a partir de un conocimiento adecuado de la realidad (es decir, que corresponda a la misma 
naturaleza de las cosas) (Pieper, 1974), la persona tendrá la potestad de elegir el bien verdadero, aunque muchas veces ello implique contrariar en algo lo que «quiere o desea» en determinada circunstancia.

La voluntad - potencia espiritual - y la afectividad — potencia sensitiva - de la persona están estrechamente relacionadas, así, «cuanto más intenso es el apetito del fin, más afectivo se hace el apetito de los medios que conducen al fin» (Echavarría, 2016, p. 61). En ese aspecto, vemos con claridad la unidad con la que opera el ser humano, en donde la intensidad de ciertos actos sensitivos da lugar a que la razón y la voluntad se nublen, y también cómo los actos de la voluntad manifestados en una intensidad muy fuerte influyen sobre el apetito sensitivo, el cual termina participando del movimiento de la voluntad (Echavarría, 2016).

La razón y el querer están estrechamente unidos y son aquellos en los que se basa la posibilidad de elección del ser humano. En este sentido, cuando el hombre elige algo, participa la razón que juzga y la voluntad que quiere; es así que se lleva a cabo una acción integrada y correspondiente a su misma naturaleza humana, acorde con sus inclinaciones naturales, puesto que, por naturaleza, el ser humano tiene una inclinación hacia la verdad y el bien; «es un instinto espiritual (instinctus rationis, dirá Santo Tomás), que inspira secretamente todas las elecciones» (Pincakers, 2007, p. 451). Por ello, podemos afirmar que cuando la elección del hombre se desvía de esta inclinación hacia la verdad y el bien, la acción ya no es una acción libre propiamente dicha. Como nos dice Santo Tomás: «que el libre albedrío pueda elegir entre diversas cosas según el orden del fin depende de la perfección de la libertad; pero que elija una cosa desviándose del orden del fin, lo que es pecar, depende de una deficiencia de la libertad» (Tomás de Aquino, 2001, I-I, q.62, a.8, ad 3).

Un aspecto importante a considerar en los actos libres de las personas es la connotación moral que estos traen consigo. Todas las decisiones y elecciones libres que lleva a cabo la persona, implican una plena conciencia sobre la realidad, sobre sí mismo y, por lo tanto, sobre lo que está haciendo, en donde se debe considerar cómo, por qué y para qué lo hace. Esta plena conciencia de todos estos factores que están inmersos en la acción libre de las personas supone una responsabilidad del ser humano acerca de su accionar. En esta línea, podemos afirmar que si bien la libertad se basa en las inclinaciones naturales del hombre, las acciones libres no son una respuesta ciega o automática frente a dichas inclinaciones, sino que entrañan una toma de posición frente a las mismas y frente a la realidad que lo circunda. Es por ello que los actos que lleva a cabo el hombre, al ser fruto de un discernimiento y elección, conllevan en sí mismos una responsabilidad, puesto que esta inclinación natural hacia la verdad y el bien requiere de una comprensión y adherencia plena por parte de la persona, y es ahí en donde sus acciones son totalmente libres. 
Incluso las manifestaciones de las pasiones pueden tener una connotación de bien o mal moral, debido a la posibilidad de su gobierno racional; por eso, «las mismas pasiones, en cuanto voluntarias, pueden decirse buenas o malas moralmente» (Tomás de Aquino, 2001, I-II, q.24, a.1). Por ello, no podemos afirmar que las pasiones de por sí sean buenas o malas, sino que al estar regidas por la razón y ponerse de manifiesto gracias a la voluntad de la persona, su bondad o malicia dependerá del gobierno que esta tenga sobre las mismas.

\section{Educación de la libertad}

La propuesta central del presente trabajo plantea que la comprensión y adherencia plena hacia el bien y la verdad, si bien está inscrita en las inclinaciones naturales espirituales del hombre, requiere ser guiada y conducida hacia su perfección, es decir, educada. Esta propuesta se basa en la definición de educación dada por Santo Tomás de Aquino «conducción y promoción de la prole al estado perfecto del hombre en cuanto hombre, que es el estado de virtud» (Martínez, 2002, p. 182). En este marco, vemos que el ser humano requiere de otro que lo guíe hacia el pleno gobierno de sí mismo, lo cual será posible por medio del logro de la virtud.

Pieper (1974) nos dice que «las virtudes nos capacitan para seguir de modo conveniente las inclinaciones naturales que pertenecen a la ley natural» (p. 77). A partir de estas consideraciones, podemos ver que la acción educativa cumple un papel fundamental, ya que, para que la persona pueda tener la capacidad para elegir el bien verdadero y no se deje llevar por sus deseos inmediatos o por la presión externa, se requiere de un gobierno personal, el cual será posible de desarrollar por medio de la forja de virtudes, especialmente las morales.

Las cuatro virtudes morales o cardinales, llamadas así porque «sobre ellas, como quicios, gira y descansa toda la vida moral humana» (Royo, 2012, p. 136), son: prudencia, justicia, fortaleza y templanza. Cada virtud se encarga de perfeccionar una potencia en particular, de modo que estas puedan llevar a cabo sus actos de la manera más acorde a su misma naturaleza (Echavarría, 2015). Es importante tomar en consideración que, para alcanzar un estado en el que la persona pueda optar libremente por el bien y la verdad y ya no necesite de ninguna guía u orientación de terceros, se debe dar un orden y jerarquía, lo cual está previamente establecido por la misma naturaleza del ser humano y el fin al que está ordenado (Echavarría, 2013). En este aspecto, las potencias que pertenecen al apetito sensitivo son aquellas que requieren, en primer lugar, de una acción educativa, para, posteriormente, pasar a la rectitud de la voluntad y la claridad del entendimiento (Amado, 2010). 
Sin embargo, dicho orden no es rígido, dado que la realidad del ser humano es tan compleja que su educación no se puede dar como si el hombre estuviera compuesto por estancos separados, antes bien, siempre se debe tener en cuenta la unidad del ser humano (Diez Canseco, 2018). A este respecto, y aunque parezca contradictorio, es sabido que la rectificación del apetito concupiscible e irascible se logra gracias al gobierno racional y voluntario; para lo cual, es necesario el perfeccionamiento de la razón y la voluntad. Es así que nos encontramos frente a un círculo - virtuosoen donde el logro de una virtud permite el logro de otra. La educación moral de la persona consiste en la forja de virtudes que permitan que se evidencie una armonía tanto en el sentir como en el pensar y actuar humanos.

La prudencia, al ser la «madre de todas las virtudes» (Pieper, 2007, p. 33), es aquella que debe estar presente para que se puedan desarrollar todas las demás, sin embargo, es también la virtud que requiere de la presencia de las demás para su plena expresión y para la posibilidad de su desarrollo. Para ser prudente es necesario poder ejercer un gobierno y moderación sobre los placeres sensibles, hacer un esfuerzo por vencer las dificultades y buscar el bien de terceros en función de lo que por justicia les pertenece. En relación con esto, vemos que estamos frente a un círculo que parece no tener principio ni final; sin embargo, dicho principio se encuentra en la prudencia de los padres (Martínez, 2002), quienes con su ejemplo y consejos muestran a sus hijos el camino a seguir para el desarrollo de virtudes, sobre todo, el de la prudencia. Tomando en consideración esta premisa fundamental, se propone una serie de etapas para la educación de las virtudes (Diez Canseco, 2018; Roqueñi, 2008).

\section{Primera etapa: moderar los placeres}

La templanza es la virtud que se encarga de la «moderación o atemperación impuesta por la razón a los actos humanos o a los movimientos pasionales, [...] aparta al hombre de aquello que le atrae en contra de la razón» (Tomás de Aquino, 2001, II.II, q.141, a.2). Esta virtud no consiste en una represión de los placeres sensitivos, sino en la regulación de los mismos por la razón; se encarga de poner orden interior al hombre, lo que permite que pueda aproximarse a la realidad con una mayor objetividad, de ahí su gran importancia en el obrar libre del ser humano.

La edad ideal para la forja de esta virtud es entre los siete primeros años de vida (Roqueñi, 2008), puesto que esta es la que se encarga de perfeccionar el apetito concupiscible, que es el que opera con más fuerza a esta edad. El lugar por excelencia para la forja de esta virtud es la familia (Palet, 2007); los padres son quienes deben procurar las condiciones necesarias para que el niño actúe de acuerdo con sus inclinaciones naturales - buscando más bien la moderación de las mismas-y no se oponga a ellas (Tomás de Aquino, 2001, II-II, q.141., a.1, ad.1). 
Son variados los hábitos a desarrollar para la forja de esta virtud, pero, en líneas generales, se debe empezar por el orden que el niño debe acostumbrarse a tener en cuanto a sus cosas, como, por ejemplo, juguetes, vestidos (ornato exterior), el orden en cuanto al trato con los demás (buen orden). Así mismo, será muy necesaria la moderación en las actividades lúdicas (eutrapelia), en la ingesta de alimentos (abstinencia), bebidas (sobriedad), en la propia excelencia (humildad), en las ansias de conocimiento (estudiosidad), en las manifestaciones de ira (mansedumbre), así como en los placeres sexuales (pudor, continencia, castidad) (Tomás de Aquino, 2001, II-II, q.141 a 170). Como vemos, moderar las manifestaciones de todos estos aspectos que tienen que ver con la rectitud en la vivencia de los placeres ayudará a que la persona pueda tomar decisiones futuras de acuerdo a lo que su razón le manda y no en función de lo que sus apetencias le reclaman; ello dará lugar a que dichas decisiones sean más autónomas y libres.

\section{Segunda etapa: vencer y resistir los obstáculos}

La fortaleza es la virtud que perfecciona el apetito irascible, y consiste en una «firmeza de ánimo para afrontar y rechazar los peligros en los cuales es sumamente difícil la firmeza» (Tomás de Aquino, 2001, II-II, q.123, a.2). Esta virtud es necesaria para que la persona pueda hacer un recto ejercicio de su libertad, dado que implica una decisión libre el hecho de tomar ciertas medidas para atacar o resistir las dificultades que se puedan presentar en el logro de los bienes elegidos.

En el niño, a partir de los siete años, ya empieza a actuar la potencia irascible que, al iniciar su movimiento y operación, produce varios e importantes cambios, esto se debe a que el niño amplía sus criterios de tiempo y espacio, y comienza a considerar los bienes futuros y no solo los presentes (Roqueñi, 2008). Por ello es que, ante la manifestación de la potencia irascible que tiende al bien arduo, se hace necesaria la forja de la virtud de la fortaleza, la cual es aquella que se encarga del fortalecimiento del ánimo para poder vencer los obstáculos que retraen a la voluntad de seguir el dictado de la razón en aras de la realización del bien (Palet, 2007).

El papel que cumplen los padres en la forja de esta virtud es muy importante, ya que ellos, con su ejemplo y acompañamiento, son el cimiento que el niño requiere para poder desarrollar la confianza y seguridad en sí mismo tan necesaria para esta virtud (Diez Canseco, 2018). La dinámica de la fortaleza consiste en la capacidad de resistir o atacar — según sea el caso- los obstáculos y dificultades que se puedan presentar en el camino al logro de las metas planteadas. La persona va preparándose para un adecuado ejercicio de su libertad futura en la medida en que sea capaz de vencer y/o resistir dichos obstáculos. Por ello, los padres y maestros deben ayudarlo en el logro de ciertos hábitos, tales como: la confianza en sí mismo y en los demás, 
indispensable para que la persona pueda tener el ánimo listo para hacer frente a las adversidades; la magnificencia, por medio de la cual el niño es capaz de llevar a cabo esfuerzos por realizar una obra bien hecha; la paciencia, que se encarga de erradicar la perturbación de la tristeza para que la persona pueda encontrarse con un ánimo tranquilo frente a las dificultades y las soporte sin desesperarse, y la perseverancia, que ayuda a que la persona sea constante en la decisión tomada hasta que, luego de mucho esfuerzo, alcance la meta planteada (Tomás de Aquino, 2001, II-II, q.128, a. un).

\section{Tercera etapa: hacer el bien al prójimo}

Otra virtud necesaria para ayudar a la persona en el pleno desarrollo de su libertad es la justicia, que rectifica a la voluntad (Tomás de Aquino, 2001, II-II, q.58, a.4). La justicia consiste en «cierta rectitud del alma por la que el hombre obra lo que deben en cualquier materia» (Tomás de Aquino, 2001, I-II, q.61, a.4) y de manera «constante y con perpetua voluntad da a cada uno su derecho» (Tomás de Aquino, 2001, II-II, q.58, a.1).

Un aspecto fundamental a considerar en la virtud de la justicia es que ordena al hombre, pero en relación al otro. Es decir, gracias a esta virtud, la persona está en capacidad de reconocer al otro en cuanto tal y se encuentra en posibilidad de respetarlo aun cuando no lo ame (Pieper, 2007), esto es, incluso cuando no exista un lazo afectivo con la otra persona, lo que naturalmente daría lugar a que se le respete y tenga cierta consideración. Es necesario destacar que esta virtud solamente se realiza respecto a otras personas (Isaacs, 1996).

El lugar natural en donde la persona lleva a cabo acciones en las que deba mostrar consideración y respeto por otros es la familia. Por medio de las normas de convivencia que se establecen en la dinámica familiar, la persona va aprendiendo el respeto a la intimidad de los demás, la consideración de su dignidad y todo aquello que haga posible una convivencia armoniosa y saludable (Diez Canseco, 2018).

Esta virtud es muy importante y esencial para la forja de la libertad plena del hombre. Se pone en evidencia un acto de plena libertad y desprendimiento cuando la persona opta en aras de procurar un bien para un tercero, aunque este no sea alguien a quien se ame con un auténtico amor de amistad, sino que la acción se lleve a cabo porque se reconoce en justicia lo que le corresponde (Pieper, 2007; Palet, 2000).

De esta forma, educar a la persona procurando el desarrollo de la justicia ayudará mucho en el reconocimiento de la realidad, dado que, en primer lugar, se requiere tener mucha claridad en cuanto a lo que en determinada circunstancia es lo co- 
rrecto hacer o dejar de hacer en función de las necesidades y de lo que en realidad corresponde a los demás. Así mismo, se requiere de mucho dominio de sí para renunciar voluntariamente a algo que se sabe que corresponde a otro. La decisión para hacer esto no es sencilla, requiere de un desprendimiento tanto hacia lo material como hacia los propios deseos y gustos en beneficio de otra persona, y para esta rectitud plena en el obrar libre del ser humano, se requiere una preparación que toma tiempo y esfuerzo, es decir, una acción educativa (Martínez, 2002). Para crecer en esta virtud, es necesario que los educadores promuevan la forja de ciertos hábitos que ayuden en el reconocimiento de la dignidad y necesidades de los demás, y moderen el trato que se debe dar entre las personas para que nunca se deje de respetar y tener en consideración las necesidades del prójimo.

Los hábitos a desarrollar son: piedad, por medio de la cual la persona cumple con sus obligaciones y da culto a sus consanguíneos; la observancia, que consiste en el respeto a las personas que son consideradas dignas en la sociedad; la dulía, que versa sobre las manifestaciones externas de honra y consideración hacia los demás; la obediencia, que es aquella parte de la justicia que consiste en seguir las indicaciones de un tercero y hacer su voluntad, pero no como un seguimiento ciego, sino a partir de una comprensión de la norma que da lugar a una decisión libre por seguirla; el agradecimiento, que es también parte especial de la justicia, por medio del cual se hace un esfuerzo por devolver de alguna manera los bienes recibidos; la veracidad, consistente en decir la verdad y ser sinceros con el otro, es decir, actuar de acuerdo con la realidad misma de las cosas; la afabilidad, que consiste en ser amable con las personas, lo que indefectiblemente va a dar lugar que las relaciones con los demás sean agradables y placenteras; la liberalidad, que se aplica principalmente al hecho de ser generoso con los bienes materiales y compartirlos con los demás, a partir del buen uso que se hace de los mismos, y, finalmente, la religión, que contempla los actos de justicia que el hombre debe a Dios, en donde debe procurar darle lo que le corresponde, honrándolo y rindiéndole culto (Tomás de Aquino, 2001, II-II, q.101 a 122).

\section{Cuarta etapa: decidir acorde a la razón}

La virtud de la prudencia, al ser considerada como la «madre de las virtudes morales» (Pieper, 2007, p. 33), es el punto de partida necesario para el desarrollo de las demás virtudes morales. En este marco, podemos decir que «la prudencia en cuanto ordenadora de todas las virtudes tiene como modo el ordenar» (Echavarría, 2013, p. 38). Esta virtud pertenece a la «facultad cognoscitiva [...] y radica propiamente en el entendimiento» (Tomás de Aquino, 2001, II-II, q.47, a.1) y no en el apetito, como las demás virtudes morales. Sin embargo, no versa solamente sobre el entendimiento de la realidad, sino especialmente sobre el obrar humano, es por ello que se cuenta también entre las virtudes morales. 
Es necesario tomar en consideración que la prudencia, además de ser virtud moral, es también virtud intelectual, y se distingue de las virtudes morales "por la distinta modalidad de objeto que especifica las potencias, ya que radica en el entendimiento, y las virtudes morales en la voluntad» (Tomás de Aquino, 2001, II-II, q.47, a.5). La prudencia es sumamente importante para que el hombre pueda llevar una vida recta, puesto que se encarga de aplicar «los principios universales a las conclusiones particulares del orden de la acción» (Tomás de Aquino, 2001, II-II, q.47, a.6). En este aspecto, se encarga de regular las acciones del hombre en función de la verdad de las cosas, disponiendo de los mejores medios para el logro del fin; por eso es que corresponde tanto al entendimiento como al apetito.

La virtud de la prudencia permite que el hombre tenga «cierta rectitud de discreción en toda clase de actos y de materias» (Tomás de Aquino, 2001, I-II, q.61. a.4), dado que la prudencia consiste en la capacidad de «deliberar, juzgar y ordenar los medios para llegar al fin debido» (Tomás de Aquino, 2001, II-II, q.47, a.10). De este forma, podemos afirmar que los actos propios de la prudencia son: «la deliberación recta, el juicio recto y — sobre todo- el recto mandato concreto» (Pieper, 1974, p. 79).

Cuando la persona posee esta virtud, podemos decir que actúa a plenitud con total libertad, debido a que ha logrado tener una comprensión profunda de la realidad con respecto a las verdades universales y no solo a lo concreto y particular; $y$, a partir de ello, está en la capacidad de aplicar dichos principios universales - los cuales son inamovibles- a cada situación particular de su vida cotidiana. En este sentido, quien decida ser guiado por esta virtud actuará siempre rectamente, porque lo hará acorde al bien de la razón.

Para la educación de la prudencia, es necesario que los educadores tomen en cuenta las partes cuasi integrales (Tomás de Aquino, 2001, II-II, q.49) de la misma, para así poder saber el camino a seguir y ayudar a su desarrollo. En primer lugar, está la memoria, que atesora todas las experiencias vividas que en algún momento permitieron una acción prudente, por ello será necesario que estas experiencias positivas sean frecuentes; luego, la inteligencia, que es aquella por medio de la cual la persona llega a tener un conocimiento de los primeros principios que rigen la realidad; la docilidad, que consiste en la disposición del niño a seguir los consejos y orientaciones de aquellos que ya están avanzados en la virtud, para así poder tener más nociones a considerar en el momento de tomar sus propias decisiones; después tenemos la sagacidad, que es aquel hábito por el que la persona sabe encontrar rápidamente lo que conviene, lo que le permite decidir y actuar inmediatamente frente a circunstancias que así lo requieran; la razón, que también forma parte de la prudencia, y, en este sentido, consiste en la capacidad de llevar a cabo razonamientos lógicos para poder encontrar el mejor camino a seguir antes de obrar; la previsión, que se encarga de ordenar los medios a seguir, de tal manera que tengan 
una jerarquización de acuerdo con la finalidad buscada; la precaución, que se encarga de ayudar a la persona a estar prevenida para evitar el mal, si es que en alguna circunstancia se encuentra presente; finalmente, la circunspección, que es aquel hábito por el que la persona actúa en función de las circunstancias que le toca vivir, por lo que cada decisión no es siempre la misma, sino que depende del momento, lugar y situación concreta que toque vivir.

La prudencia es la virtud que, por su misma naturaleza, está más estrechamente relacionada con las acciones libres del ser humano. No será posible una acción libre realmente si la persona no es prudente, puesto que esta virtud es la que la habilita para una plena comprensión de la verdad de las cosas y para un recto obrar en cada circunstancia concreta de su vida; y la libertad justamente consiste en dicha posibilidad.

La familia es el lugar en donde la persona aprende -mediante el ejemplo de sus padres - a ser prudente; sin embargo, Isaacs (1996) nos dice que la forja de esta virtud en el seno familiar no es tarea sencilla: «la vida familiar exige una actividad continuada. Esta actividad dificulta el proceso de reflexión y, como consecuencia, existe una tendencia a reaccionar frente a las situaciones nuevas que van surgiendo, más que a afrontarlas con serenidad para tomar decisiones acertadas» (p. 337). Para el logro de esta virtud, es necesario un largo camino, dado que esta no es fácil de alcanzar, requiere de cierta madurez tanto a nivel cognitivo como en el obrar; una vez alcanzada, la persona está en capacidad de optar libremente por el bien.

\section{Libertad moral}

El fin de la educación de la persona humana debe ser que esta alcance la capacidad de tomar por sí misma las decisiones más acertadas que la lleven al bien según el máximo despliegue de sus potencialidades, es decir, que dichas decisiones estén acordes a lo propio de la naturaleza humana, a la verdad y al bien de la realidad. Cuando la persona alcanza esta capacidad, podemos afirmar que ya no requiere de otro que la guíe y conduzca hacia el bien, decimos así que ha pasado de su estado de niño a un estado adulto (Martínez, 2002). En este punto se da un discernimiento de la realidad en conformidad con la razón y, por ende, la toma de decisiones siempre se llevan a cabo en aras de alcanzar el bien que la razón le muestra. Dichas decisiones implican un pleno ejercicio de la libertad en la persona, puesto que se da un despliegue de sus potencialidades de acuerdo a lo que están llamadas a ser, pero basado en lo que libremente la persona decide, sin coacción alguna.

Como es sabido, toda decisión libre implica una responsabilidad, ya que la persona es capaz de asumir como propias sus decisiones, rigiendo su vida por sí misma y 
encontrándose en condiciones de hacerse íntegramente responsable de la misma (Millán Puelles, 1989); en este contexto, podemos hablar de una libertad moral.

Como hemos venido planteando a lo largo del trabajo, en la persona, el logro de este pleno ejercicio de la libertad proviene del desarrollo de virtudes, especialmente de las virtudes morales y, en particular, de la prudencia. La prudencia imprime una perfección en el obrar del hombre, es así que el prudente es aquel que evalúa cada circunstancia particular que le toca vivir y, a partir de esto, elige el mejor camino a seguir para alcanzar el bien al que tiende. Estas decisiones son justamente las decisiones libres que lleva a cabo el hombre y que, a medida que se van arraigando en su ser, lo hacen cada vez más libre e íntegramente responsable de sus acciones (Millán Puelles, 1989). Planteamos esta noción de libertad desde la concepción de libertad de calidad, en donde la persona, al optar por el bien, es cuando realmente es libre, porque la opción por el mal esclaviza a la persona a sus vicios y la aleja cada vez más de aquello que responde a su propia naturaleza.

La persona auténticamente prudente no opta por el bien por seguir un convencionalismo social o guiado por la tendencia a la satisfacción de sus placeres o caprichos, antes bien, lo hace porque la virtud se encuentra plenamente internalizada en él como una «segunda naturaleza» (Tomás de Aquino, 2001, I-II, q.78, a.2). Por ello, al darse esta autenticidad y connaturalidad (Maritain, 1951) hacia las acciones buenas, es que podemos decir que el accionar es plenamente libre, acorde a la naturaleza humana; como dice Enrique Martínez (2002), la prudencia hace del hombre un ser libre moralmente, pues le da auténtico señorío sobre sus actos.

El Papa Francisco (2016) nos dice: «la libertad es algo grandioso, pero podemos echarla a perder» (n. 267), y plantea que es la vida virtuosa la que va construyendo la libertad, fortaleciéndola y educándola. Por esto es que planteamos que la libertad es posible de ser educada, y para ello es necesario el desarrollo de virtudes, las cuales no se podrán dar en la persona - de manera natural— si es que no media una acción educativa.

En este sentido, la educación consiste en una preparación de la persona para que pueda llevar a cabo toda obra buena (Martínez, 2002), un accionar plenamente libre, que se alcanza cuando la persona llega al estado adulto del que hablamos anteriormente. No podemos afirmar que este estado se alcanza en determinado momento de la vida, debido a que el hombre siempre está en constante progreso en la virtud, ya que es susceptible de crecimiento (Martínez, 2002). Es así que el hecho de llegar a este estado y poder tomar decisiones libres, acordes al bien y a la verdad de la realidad, es algo que el ser humano debe procurar poner en práctica en cada circunstancia concreta de su vida, por lo tanto, no es algo ya acabado o logrado, sino que es un dinamismo que se debe actualizar en cada decisión a tomar. 
La acción educativa consiste en una conducción y promoción de la persona para que pueda alcanzar la virtud, es decir, este estado adulto que hemos venido nombrando. Por esta razón, dicha acción tiene una finalidad, pero esta no corresponde a aquello que cada educador se proponga según sus caprichos o creencias, sino que es algo que está acorde a lo propiamente natural en el ser humano: «es bastante advertir que si el fin de la actividad educativa se nos presenta como moralmente necesario, ha de ser, a la vez, algo natural, puesto que el orden de la moralidad está fundado en el de la naturaleza» (Millán Puelles, 1989, p. 53). En este sentido, llegar a este estado adulto es a lo que por naturaleza está llamado el hombre.

Con el logro de la virtud, las potencias del hombre alcanzan un estado de perfección que le confieren una especie de «condicionamiento permanente, desde el cual hace uso de su libertad» (Millán Puelles, 1989, p. 61), de tal manera que este uso sea el más conforme con la naturaleza humana; a su vez, vemos que este condicionamiento se refiere a lo que Santo Tomás denomina «segunda naturaleza». Esta habitual inclinación de la persona a responder acorde a su propia naturaleza es lo que se logra con la educación y es la única vía por la que el hombre obra verdaderamente de una manera libre, puesto que la virtud es aquello que permite que el hombre libremente opte por el bien que reclama su naturaleza. Cuando esto sucede y la persona ya no necesita de ninguna indicación o sugerencia para llevar a cabo estas acciones, podemos afirmar que ha alcanzado su estado adulto o estado de virtud.

\section{Referencias}

Amado, A. (2010). La educación cristiana (2.a ed.). Barcelona: Ediciones Scire Universitaria.

Aquino, T. de. (2001). Suma Teológica. Madrid: Biblioteca de Autores Cristianos.

Barrio Maestre, J. M. (1998). La problemática de la antropología filosófica de la educación. Revista de Educación, 316, 111-132.

Brennan, R. (1960). Psicología tomista: Análisis filosófico de la naturaleza del hombre. Madrid: Editorial Científico Médica.

Diez Canseco, M. (2018). Educación de la virtud en los ámbitos familiar y escolar. Arequipa: Universidad Católica San Pablo.

Echavarría, M. (2012). Influencias de la psicología contemporánea en las corrientes pedagógicas. En Martínez, E. (Dir.). ¿Una sociedad despersonalizada? Propuestas educativas (pp. 49-86). Barcelona: Editorial Balmes.

Echavarría, M. (2013). Templanza e Intemperancia: Actualidad psicológica de la concepción tomista de las virtudes. Recuperado el o7 de julio de 2019 de: https://www.academia.edu/4037761/Templanza_e_Intemperancia._Actualidad_psicológica_de_la_concepción_tomista_de_las_virtudes 
Echavarría, M. (2015). Prudencia, Fortaleza, Justicia y Amistad: Propuestas terapéuticas y educativas. Barcelona: Ediciones Scire.

Echavarría, M. (2016). El corazón: Un análisis de la afectividad sensitiva y la afectividad intelectiva en la psicología de Tomás de Aquino. Recuperado el o7 de julio de 2019 de: https://www.academia.edu/18581443/El_corazón_Un_análisis_de_la_afectividad_sensitiva_y_la_afectividad_espiritual_en_la_psicologí_de_Tomás_de_Aquino

Elders, L. J. (1997). La libertad humana en la construcción de la persona. En Sarmiento, A. El Primado de la persona en la moral contemporánea: XVII Simposio Internacional de Teología de la Universidad de Navarra (pp. 177-187). Navarra: Servicio de Publicaciones de la Universidad de Navarra.

Francisco. (2016). Amoris Laetitia. Lima: Editorial Paulinas.

Isaacs, D. (1996). La educación de las virtudes humanas (12.르. ed.). Navarra: EUNSA.

López, J. M. T. (1977). La relación libertad-educación: Incomprensiones en torno a ella, su sentido y su estructura fundamental. Revista española de Pedagogía, 35(136), 179-192.

Maritain, J. (1951). Sobre el conocimiento por connaturalidad. Recuperado el o7 de julio de 2019 de: http://www.jacquesmaritain.com/pdf/o3_EPI/o7_EP_ConConna.pdf

Martínez, E. (2002). Persona y educación en Santo Tomás de Aquino. Madrid: Fundación Universitaria Española.

Millán Puelles, A. (1989). La formación de la personalidad humana (7.․e ed.). Madrid: Rialp.

Ocampo, M. (2013). Tendencia natural y libre en el pensamiento de Santo Tomás de Aquino. Recuperado el o7 de julio de 2019 de: http://www.infocatolica.com/ blog/concordia.php/1502260803-tendencia-natural-y-libre-en

Palet, M. (2000). La familia educadora del ser humano. Barcelona: Ediciones Scire. Palet, M. (2007). La educación de las virtudes en la familia. Barcelona: Ediciones Scire.

Pieper, J. (1974). El descubrimiento de la realidad. Madrid: Rialp.

Pieper, J. (2007). Las virtudes fundamentales (9. ${ }^{\underline{a}}$ ed.). Madrid: Rialp.

Pinckaers, S. (2007). Las fuentes de la moral cristiana (3. - ed.). Navarra: EUNSA.

Roqueñi, J. (2008). Educación de la afectividad (2.ㄹ ed.). Navarra: EUNSA.

Royo, A. (2012). Teología de la perfección cristiana. Madrid: Biblioteca de Autores Cristianos.

Verneaux, R. (1988). Filosofía del hombre (10. ed.). Barcelona: Herder. 